\title{
Malignant Ethmoid Sinus Neoplasm
}

National Cancer Institute

\section{Source}

National Cancer Institute. Malignant Ethmoid Sinus Neoplasm. NCI Thesaurus. Code C3541.

A primary or metastatic malignant neoplasm involving the ethmoid sinus. 